\title{
puente metálico sobre el río Save
}

\author{
G. SCHAFER, ingeniero
}

$565-28$

\section{simopsis}

El primitivo puente colgante Rey Alejandro, sobre el río Save, Yugoslavia, destruido durante la última guerra mundial, ha sido reconstruido por la vía de concurso internacional, al cual se presentaron 32 proposiciones de diferentes países. La solución aceptada fue una estructura metálica de $261 \mathrm{~m}$ de luz.

El arco de este tramo tiene una sección hueca en forma de cajón. El tablero se compone de una placa ortotrópica, de la que, previamente, se han realizado varios ensayos.

La obra, de tres tramos-dos para los accesos y uno central一, se construyó por procedimientos sin andamiaje, es decir, en voladizo. La anchura de la calzada es de $12 \mathrm{~m}$, y dos andenes, de $3 \mathrm{~m}$ cada uno, para peatones. Los arcos de las vigas cajón tienen $9,60 \mathrm{~m}$ de canto sobre las pilas y $4,50 \mathrm{~m}$ en la clave. Las pendientes hacia uno y otro
lado de la clave son del 0,7 y $0,25 \%$, respectivamente. La rigidez del tablero es perfecta y sufieiente para soportar las cargas previstas y para transmitir los efectos del viento a las pilas.

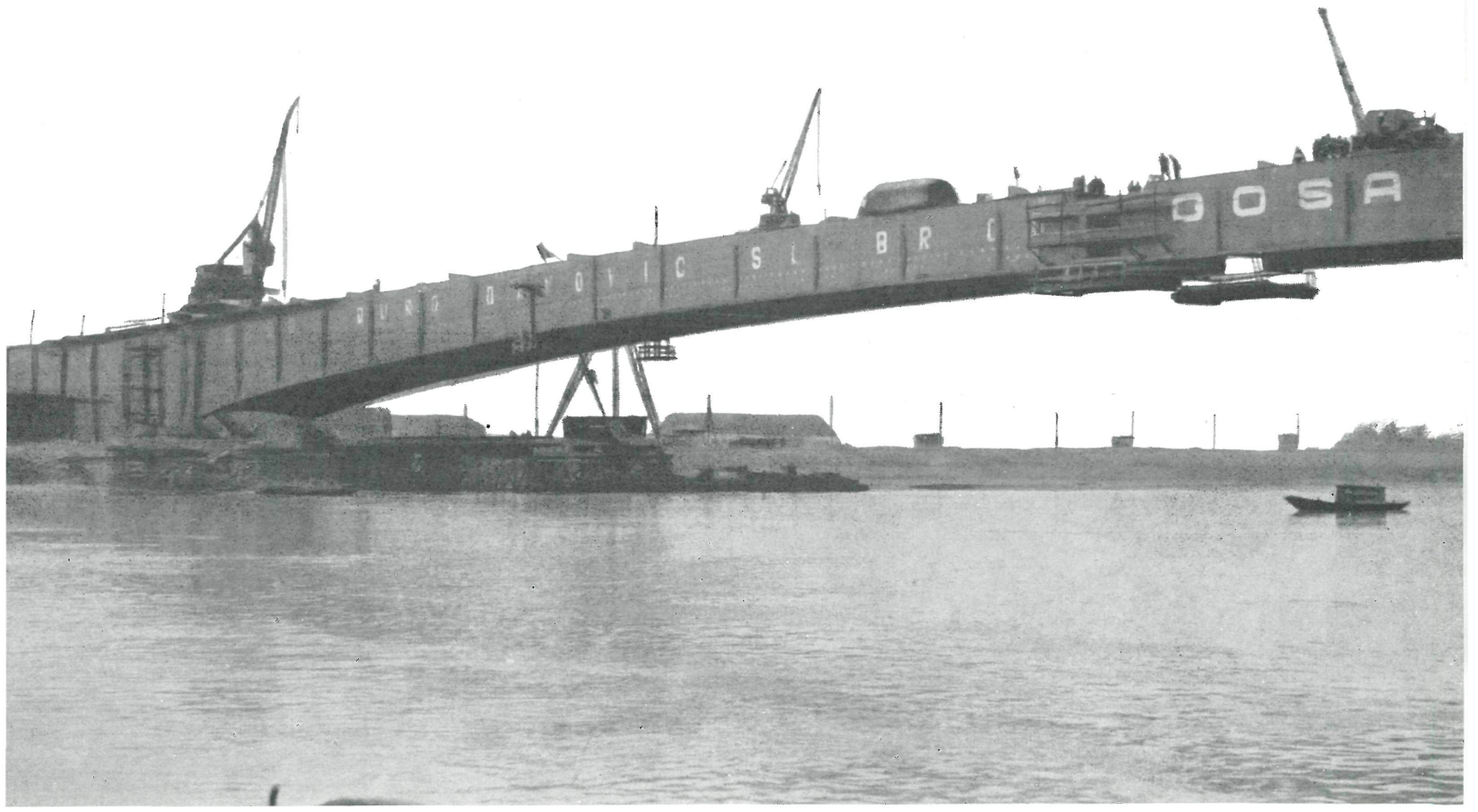




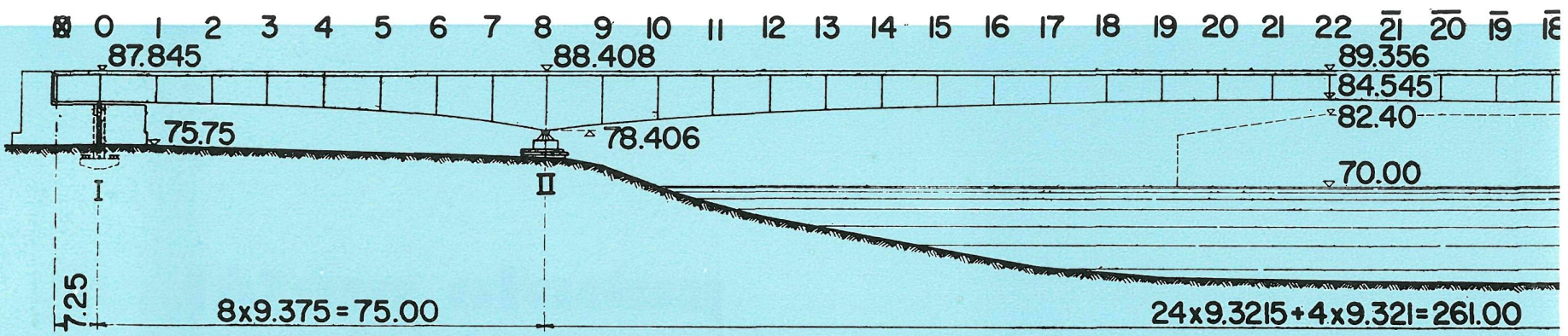

a Iza d o

pa n a

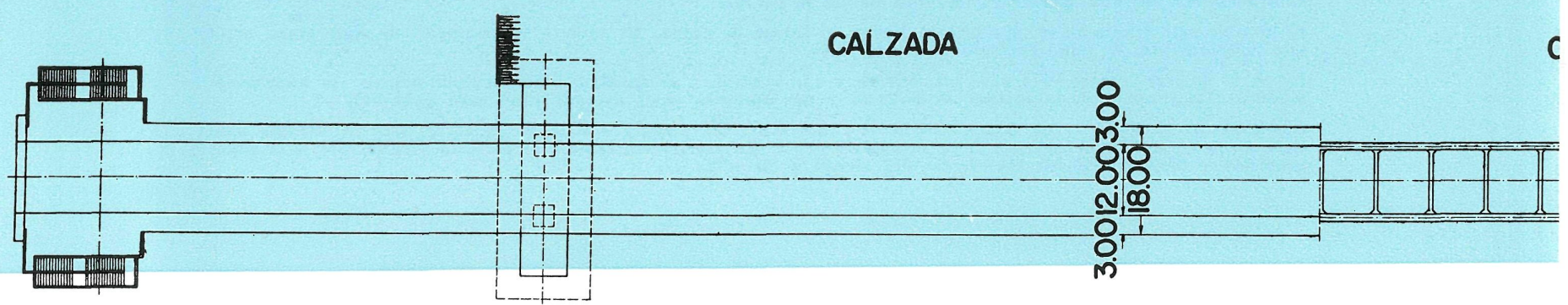

\section{intmoducción}

El puente Rey Alejandro, construido en el período 1930-34 sobre el río Save, en Yugoslavia, era de tipo colgante, y fue destruido durante la última guerra mundial.

Terminado el calamitoso período económico de la postguerra, se decidió-pues así lo aconsejaban las circunstancias-la reconstrucción de la obra. Con esta finalidad se abrió un concurso internacional para elegir el proyecto de ejecución de la reconstrucción de dicho puente, al cual se presentaron 32 proposiciones de diferentes países, entre los que se contaban firmas de gran capacidad técnica y constructiva, incluidas representaciones de casas yugoslavas.

La solución adoptada fue la de tipo metálico, con un tra mo central de $261 \mathrm{~m}$ de luz. Con este proyecto se puede reducir considerablemente el peso de obra muerta, respecto del puente colgante original. El peso propio resultó ser de 3.800 toneladas.

El arco que salva el río tiene una sección transversal en forma de cajón, y se ha proyectado teniendo en cuenta el estado actual de desarrollo y evolución de las construcciones metálicas en la industria de las empresas especializadas ce la Alemania Occidental. 

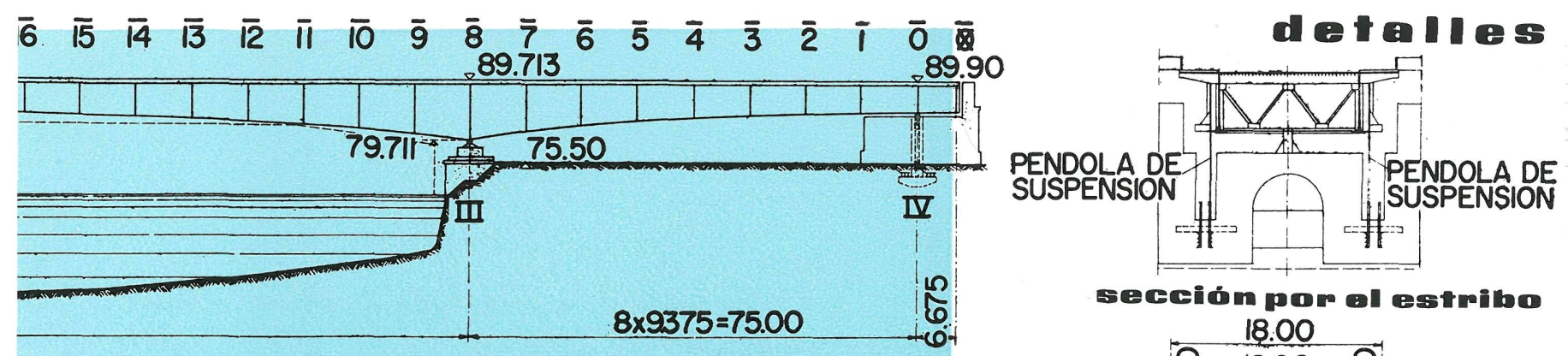

sección por al estrmibo

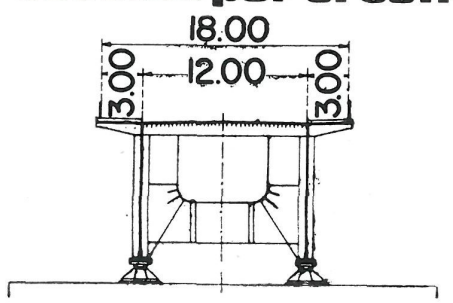

sección por ell apoyo
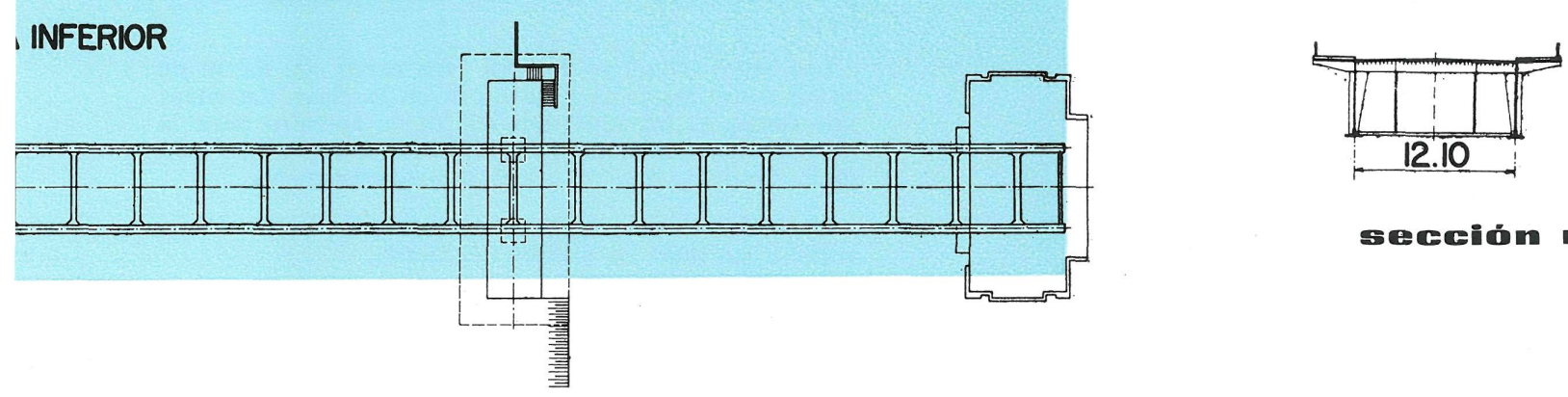

SECción mormun

Dada la luz y peso, el proyecto se comprobó por medio de ensayos en laboratorio, ya que no se disponía de precedente alguno para esta luz de $261 \mathrm{~m}$. El tablero se compone de una placa ortotrópica, de la que se hicieron repetidos ensayos para estudiar su comportamiento.

Esta luz de $261 \mathrm{~m}$, primera de las utilizadas hasta hoy, así como el intento de construir en voladizo, partiendo de los dos arranques simultáneamente, para llegar a un brazo de $130 \mathrm{~m}$ en voladizo, exigieron no pocos esfuerzos y cuidados en el cálculo estático de este tramo central.

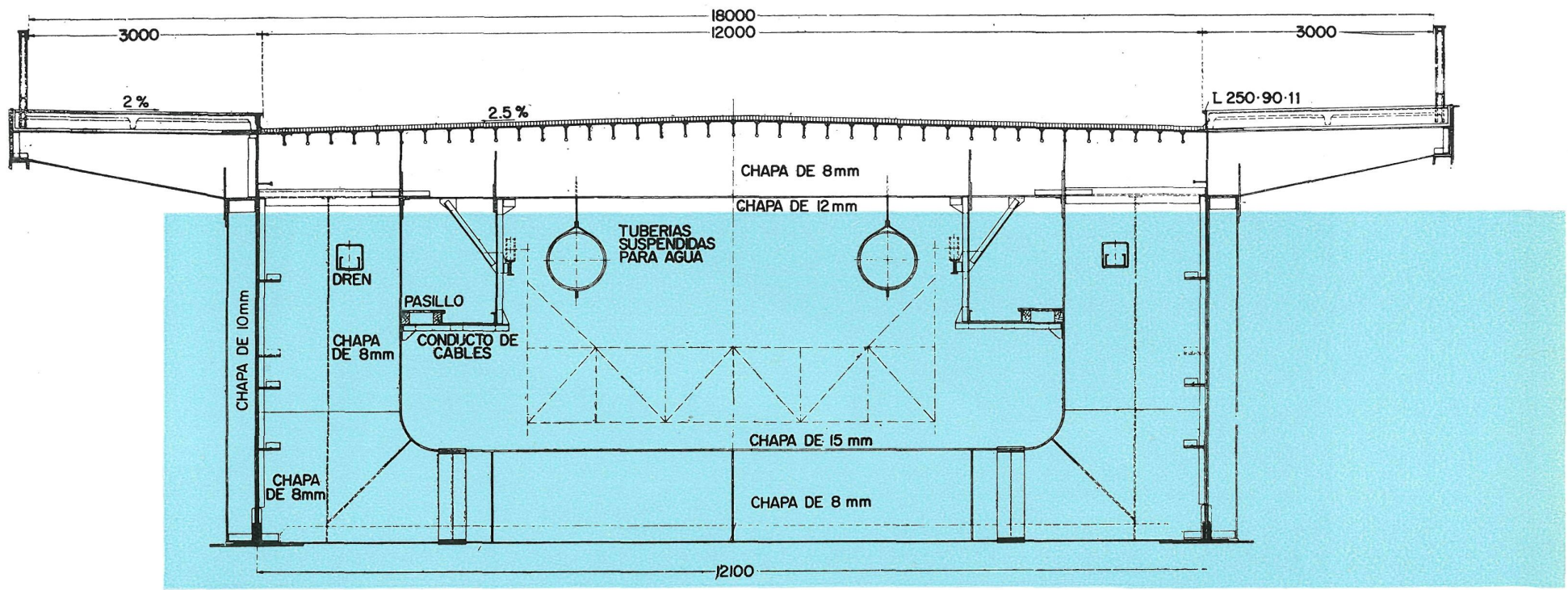




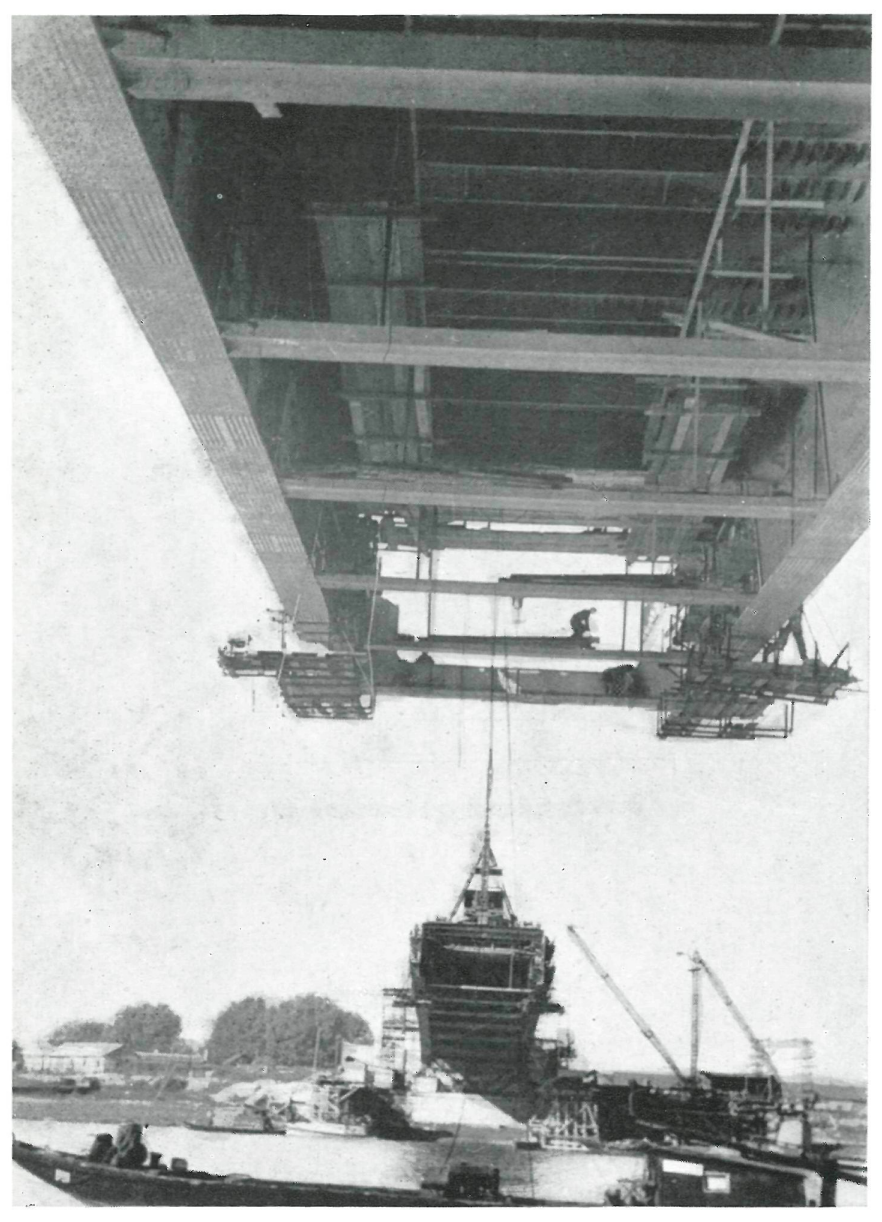

Entramado auxiliar de madera.

Arriostramiento de la cabeza interior.

El puente, recientemente terminado, presenta un mag. nífico aspecto arquitectónico, y sus cálculos fueron tan perfectcs que, durante la construcción, se pudo comprobar la exigua diferencia observada entre los valores calculados teóricamente y los reales que aparecieron en la obra.

\section{descripción de la obra}

El puente consta de tres tramos: dos de acceso, de $75 \mathrm{~m}$ de luz, uno a cada lado del central, que tiene $261 \mathrm{~m}$ de luz, coronados por una calzada de $12 \mathrm{~m}$ de anchura, y dos andenes, de $3 \mathrm{~m}$ de anchura cada uno, para peatones.

Los arcos metálicos forman viga cajón con 9,6 $\mathrm{m}$ de canto sobre las pilas y de $4,5 \mathrm{~m}$ en la clave. La curva que forma el intradós deja $150 \mathrm{~m}$ de anchura para la libre navegación fluvial. Las pendientes a uno y otro lado de la clave son del 0,75 y $0,25 \%$, respectivamente.

El tablero tiene rigidez suficiente para poder transmitir a las pilas los efectos del viento.

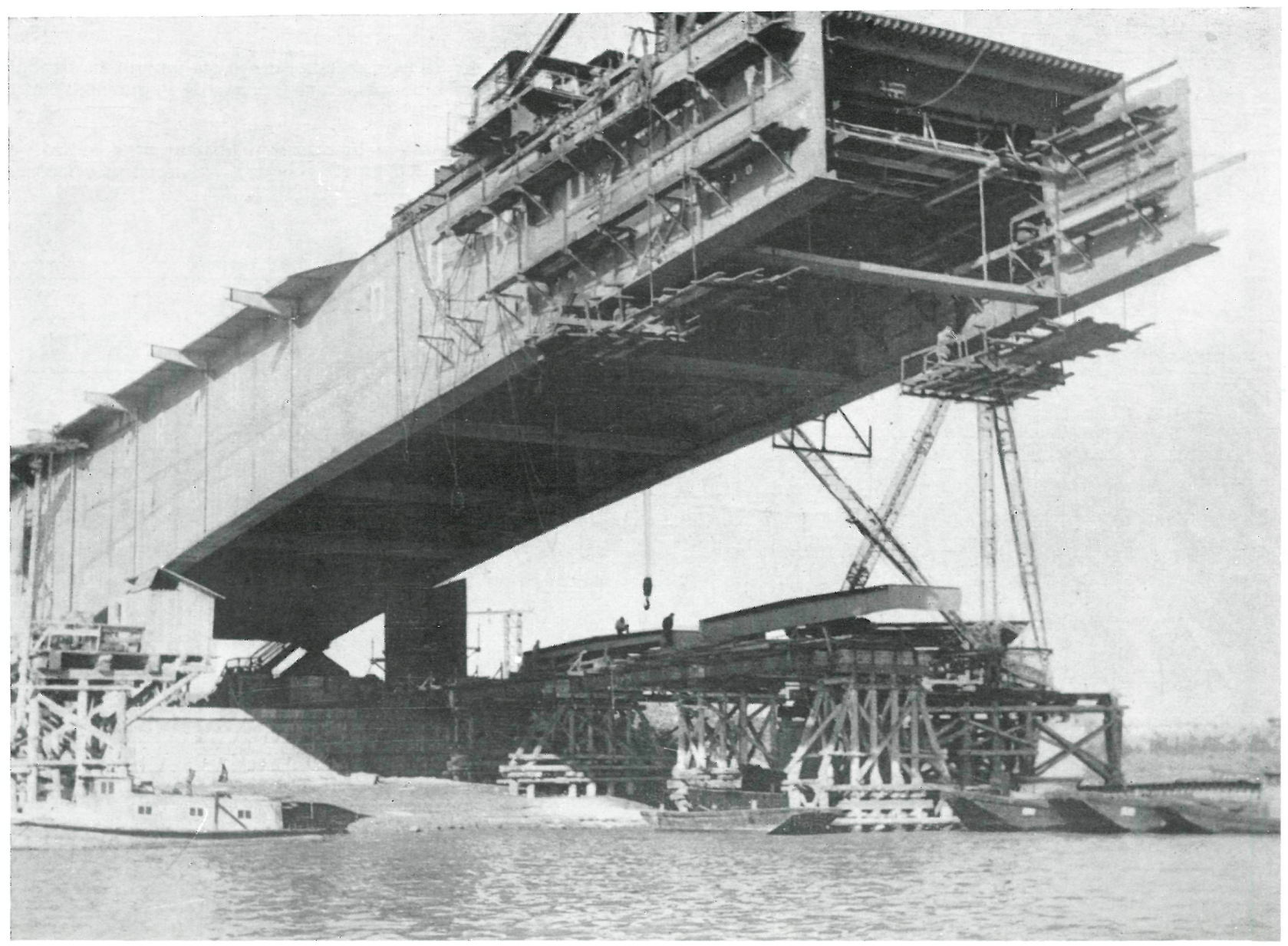




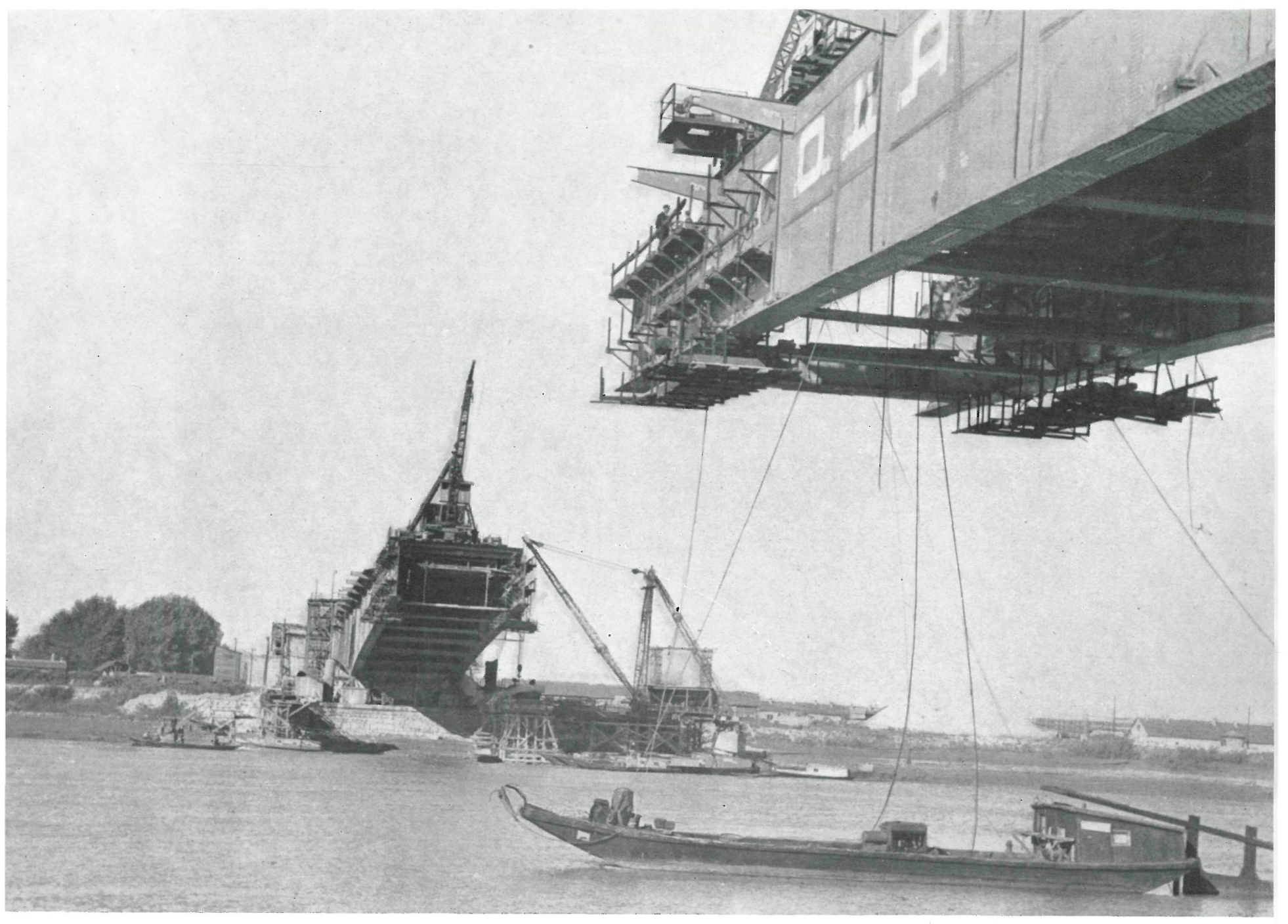

Soportes para los andenes.

Intradós del puente.

En los nudos la estructura se ha reforzado con piezas que, actuando como tirantes, arriostran las dos cabezas de las vigas maestras. En los arranques se han montado vigas transversales. Uno de los dos apoyos del tramo central es fijo, mientras que el otro es móvil. En los apoyos el puente descansa sobre péndulos, ya que en estos puntos aparecen momentos negativos debidos a las cargas estáticas y móviles. Cada viga principal origina una reacción en los apoyos de 2.550 toneladas.

La calzada cuenta con una capa asfáltica de rodadura de $5 \mathrm{~cm}$ de espesor, que en los andenes se reduce a $2 \mathrm{~cm}$. Tanto la calzada como los andenes disponen de un encintado metálico.

\section{carrgas}

Para la redacción del proyecto se tuvieron en cuenta las normas yugoslavas, que exigen una carga estática uniforme de $350 \mathrm{~kg} / \mathrm{m}^{2}$. Para el viento se ha admitido una carga de $250 \mathrm{~kg} / \mathrm{m}^{2}$ con el puente descargado, y sólo de $90 \mathrm{~kg} / \mathrm{m}^{2}$ si está cargado. Las deformaciones se han calculado con temperaturas límite de $\pm 35^{\circ} \mathrm{C}$ y una diferencial de $15^{\circ} \mathrm{C}$ entre cabezas de las vigas.

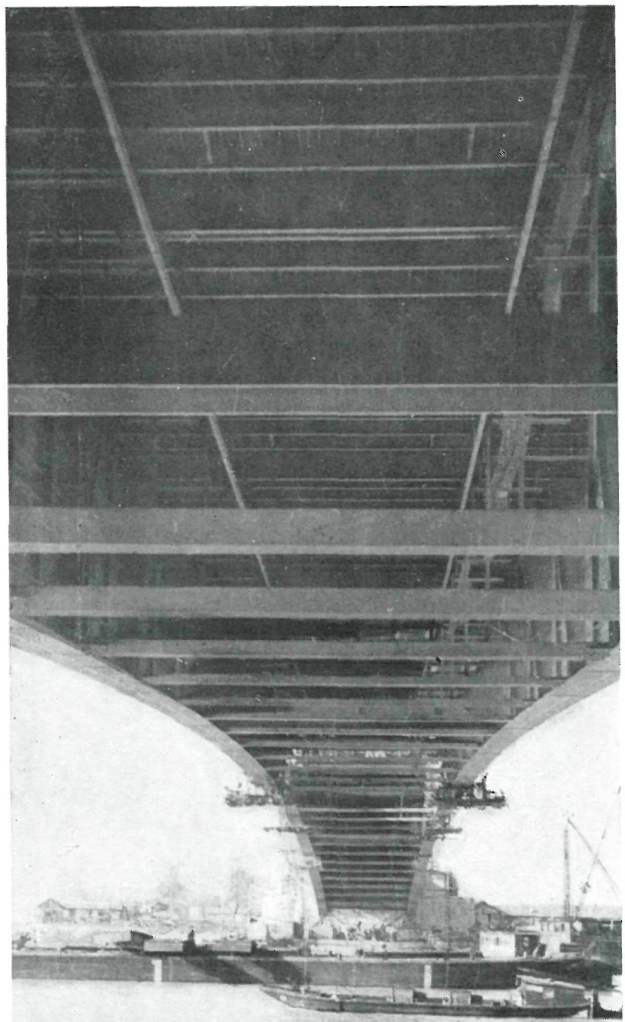



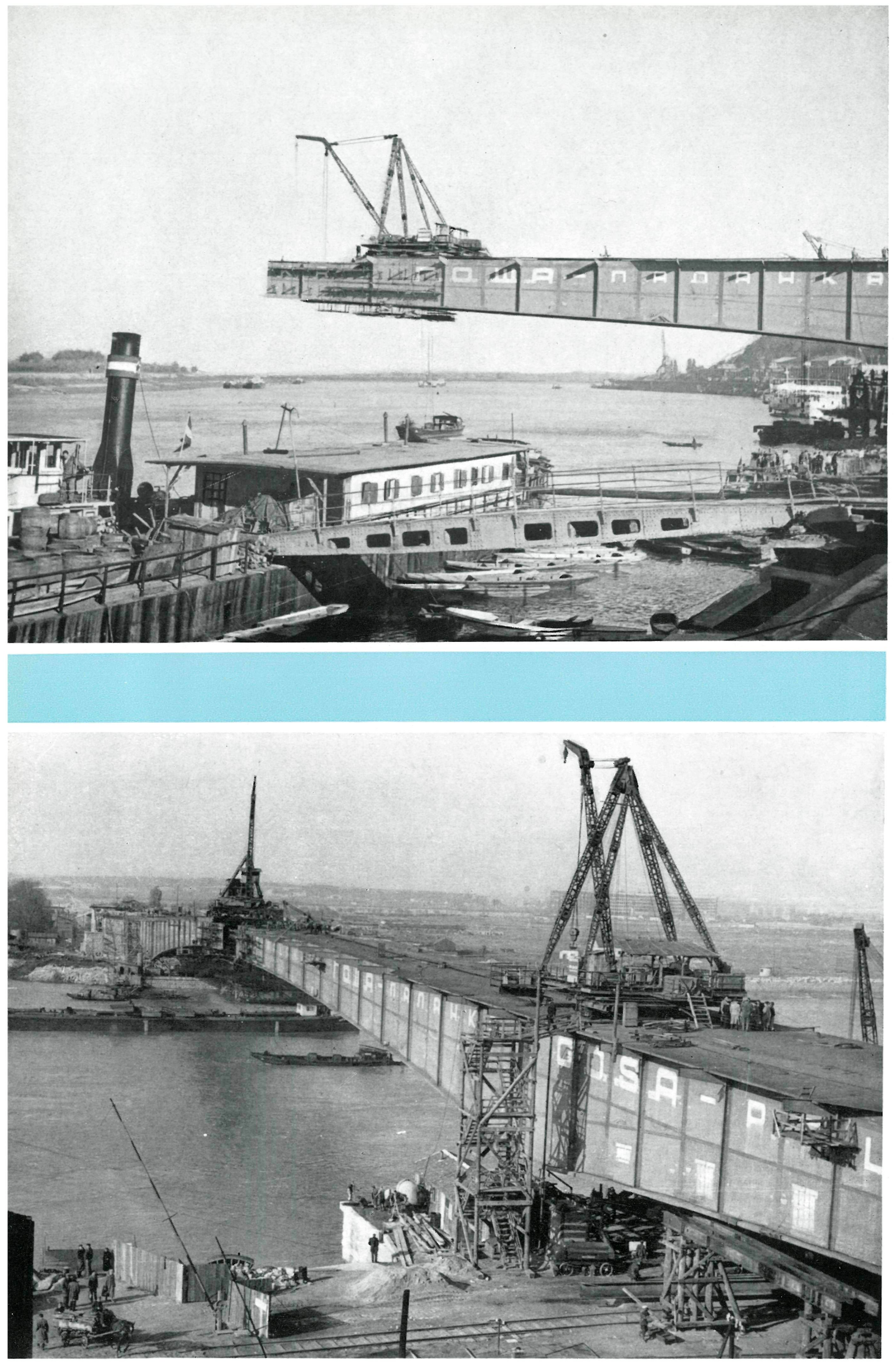


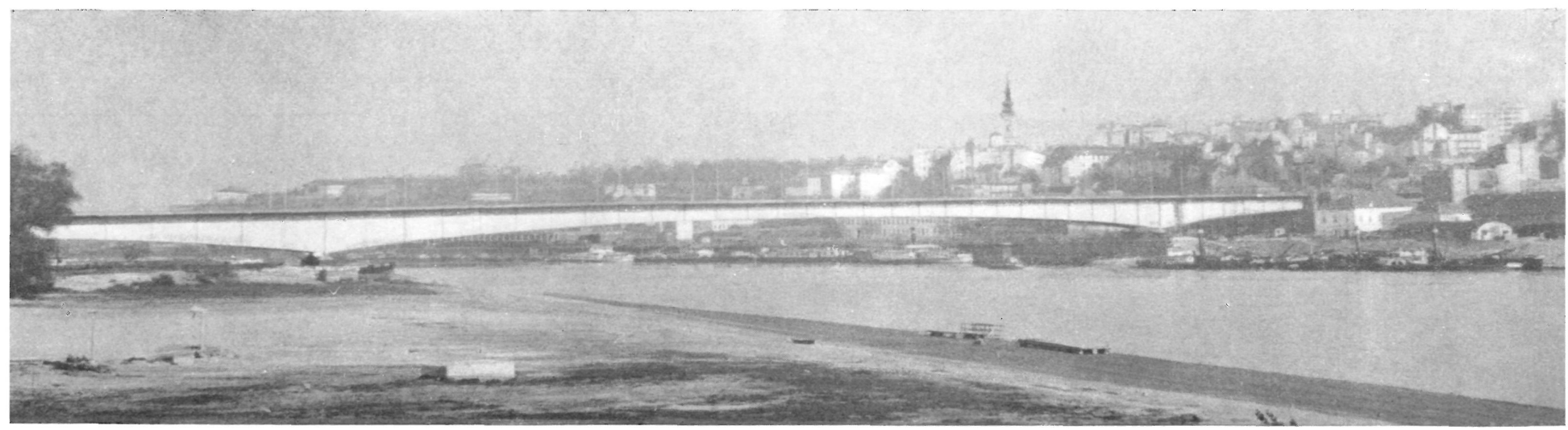

El armazón general del puente está constituido por chapa de espesor variable entre 12 y $15 \mathrm{~mm}$ y, en general, soldada, así como los elementos que forman la placa ortotrópica del tablero. Todo este armazón lleva una serie de elementos de rigidez que solidarizan el conjunto.

\section{montraje}

Los accesos se construyeron siguiendo los procedimientos ortodoxos en esta materia y, en cambio, el tramo central se montó en voladizo y sin utilizar soporte alguno auxiliar. Este último tramo se empezó, simultáneamente, partiendo de ambas extremidades opuestas para cerrar en la clave. Las vigas principales se fabricaron en Yugoslavia, mientras que el tablero ortotrópico se preparó en los talleres de la casa constructora.

Para el montaje de piezas pesadas se utilizaron grúas tipo pórtico y ordinarias, de una capacidad de 25 toneladas. Para llegar al cierre definitivo se instaló un dispositivo que permitió nivelar debidamente los dos brazos que tenían que unirse para formar un solo arco.

El puente terminado.

Tramo central cerrado.

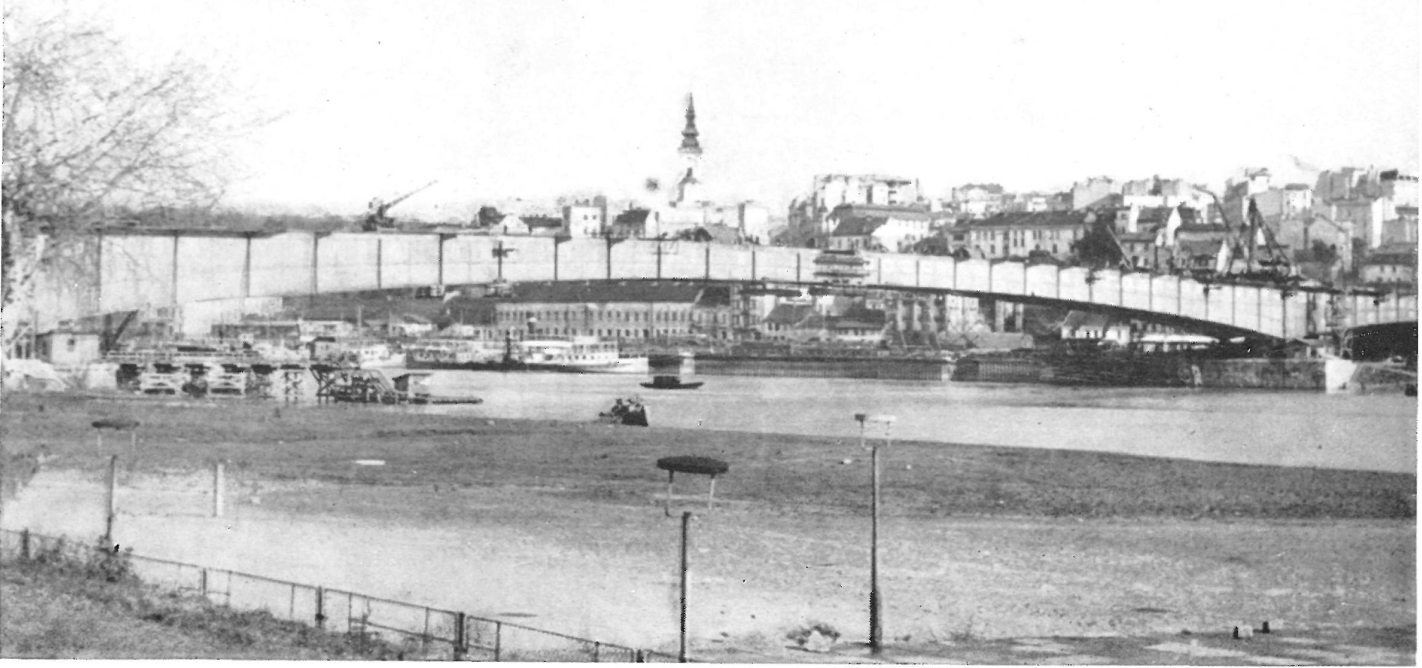




\title{
Pont unétrallique sur le Save
}

G. Schäfer, ingénieur.

L'ancien pont suspendu, Roi Alexandre, sur le Save, en Yougoslavie, détruit pendant la dernière guerre mondiale, a été reconstruit par la voie d'un concours international auquel ont été présentés 32 projets de différents pays. La solution qui a été choisie est une structure métallique de $261 \mathrm{~m}$ de portée.

L'arc a une section creuse en forme de caisson. Le tablier se compose d'une plaque orthotropique qui a fait l'objet de plusieurs essais.

L'ouvrage, en 3 tronçons-deux pour les accès et un central-, a été construit en encorbellement à l'aide de procédés sans échafaudage. La chaussée a $12 \mathrm{~m}$ de largeur et les deux trottoirs pour piétons, $3 \mathrm{~m}$ chacun.

Les arcs des poutres-caisson mesurent 9,60 $\mathrm{m}$ de hauteur sur les piles et 4,50 $\mathrm{m}$ à la clé. Les pentes vers l'un et l'autre côté de la clé sont de 0,7 et $0,25 \%$, respectivement. La rigidité du tablier est parfaite, suffisante pour supporter les charges prévues et pour que les effets du vent soient transmis aux piles.

\section{Metal bridge ouer the river Scuve}

G. Schäger, engineer.

The original «Kinfi Alexander» suspension bridge over the Save river, Yugoslavia, which was destroyed during the last world war has been reconstructed, after organising an international prize award for its design. 32 projects from various countries were submitted. The chosen design is a metal structure, of $261 \mathrm{~ms}$ span.

The arch of the main span has a hollow box section. The deck consists of an orthotropic slab, which has been subjected to a number of tests.

The bridge has three spans (two approach and one central span), and was built without scaffolding, i. e., by cantilevering it from the springers. The roadway is $12 \mathrm{~ms}$ wide, and there are two $3 \mathrm{~ms}$ wide footways.

The box section arch girders are $9.60 \mathrm{~ms}$ deep over the piles, and $4.50 \mathrm{~ms}$ at the crown. The slopes on either side of the crown are 0.7 and $0.25 \%$, respectively. The deck is very rigid, and is well capable of supporting the design loads and windage.

\section{Stablbrioke iuber die Scuve}

\author{
G. Schäfer, Ingenieur.
}

Die ursprüngliche Hängebrücke «König Alexander» über die Save, in Jugoslavien, die während des letzten Weltkrieges zerstört wurde, hat man auf dem Wege eines internationalen Wettbewerbes, zu dem 32 Vorschläge verschiedener Länder vorgelegt wurden, wieder aufgebaut. Die angenommene Lösung ist eine Stahlkonstruktion mit einer Spannweite von $261 \mathrm{~m}$.

Der Bogen dieses Abschnittes hat einen Hohlteil in Kastenform. Die Fahrbahn setzt sich aus einer ortotropen Platte zusammen, mit der verschiedene Versuche durchgeführt wurden.

Das Bauwerk aus drei Abschnitten, zwei für die Anfahrten und ein Mittelabschnitt, wurde im freien Vorbau-Verfahren erbaut, d. h. ohne Gerüst. Die Fahrbahn hat $12 \mathrm{~m}$ Breite und zwei Gehwege für die Fussgänger, ein jeder mit $3 \mathrm{~m}$.

Die Bogen der Hohlbalken weisen 9,60 $\mathrm{m}$ Balkenhöhe über den Pfeilern und 4,50 $\mathrm{m}$ im Scheitel auf. Die Gefälle vom Scheitel aus nach der einen und anderen Seite hin sind von $0, \%$ und $0,25 \%$ jeweilig. Die Fahrplatte hat eine vollkommene und genügende Steife, um die vorgesehenen Lasten auszuhalten und die Windwirkungen auf die Pfeiler zu übertragen. 